\section{Le statut \\ nutritionnel, \\ l'activité \\ et la condition \\ physique \\ des adolescents \\ sous influence}

\section{Résultats de l'étude \\ HELENA}

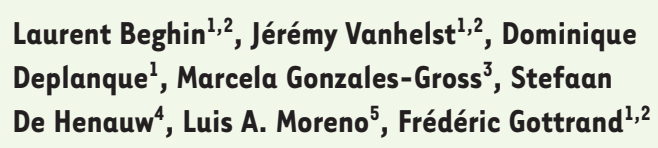

de nombreux facteurs tels que le niveau socioéconomique, le style de vie, l'environnement personnel et collectif, le profil alimentaire (incluantl'allaitement), certains polymorphismes de gènes impliqués dans la corpulence et le métabolisme, le niveau d'activité physique et la condition physique. <

L'augmentation durant ces dernières décennies de la prévalence du surpoids et de l'obésité touche toutes les tranches de la population, enfants, adolescents et adultes, aussi bien dans les pays industrialisés que les pays en voie de développement. Ce problème alarmant est devenu un enjeu de santé publique majeur [1]. L'enfance et l'adolescence sont des périodes importantes si l'on considère l'influence que peuvent avoir la nutrition, l'alimentation et l'activité physique sur le devenir de l'individu. En effet, c'est lors de la petite enfance puis de l'adolescence que la croissance est la plus forte. Les besoins nutritionnels sont alors considérablement augmentés [31] $(\rightarrow)$.

C'est également durant cette

$(\rightarrow)$ Voir la Nouvelle période que les habitudes ali- $\mathbf{m} / \mathbf{s} \mathbf{n}^{\circ} \mathbf{1}$, janvie mentaires et d'activité physique 2007, page 5 se construisent. Elles perdureront à l'âge adulte [2]. Cette période particulière favorise également les comportements alimentaires à risques et les répercussions du surpoids et de l'obésité qui auront été développées lors de l'adolescence sont nombreuses à l'âge adulte : maladies cardio-vasculaires (infarctus, accident vasculaire cérébral, hypertension) et métaboliques (principalement diabète de type 2), cancer (sein,

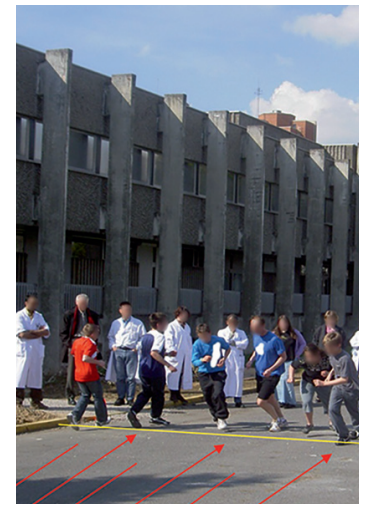

côlon, endomètre, rein, œsophage) et problèmes musculo-squelettiques (diminution de la densité minérale osseuse et augmentation des problèmes articulaires). Aussi, I'un des enjeux du XXI siècle en termes de promotion de la santé et de prévention des maladies, est de mettre en place dès l'enfance et l'adolescence des comportements et un style de vie qui soient favorables à la santé à l'âge adulte. Afin d'optimiser les stratégies de lutte contre le surpoids et l'obésité, il est donc nécessaire d'avoir un état des lieux sur l'état nutritionnel et le style de vie des adolescents, et de définir les facteurs qui les déterminent. En Europe, comme en témoigne la répartition du surpoids et de l'obésité rapportée par Lobstein et al [3], la variabilité entre les pays est très élevée. Elle découle probablement de différences socio-culturelles et de certaines inégalités de santé. L'objectif de I'étude HELENA faisant participer des adolescents de différents pays de l'Europe, était donc d'évaluer le statut, en particulier nutritionnel, de ces jeunes à un temps $t$, afin de pouvoir envisager les 


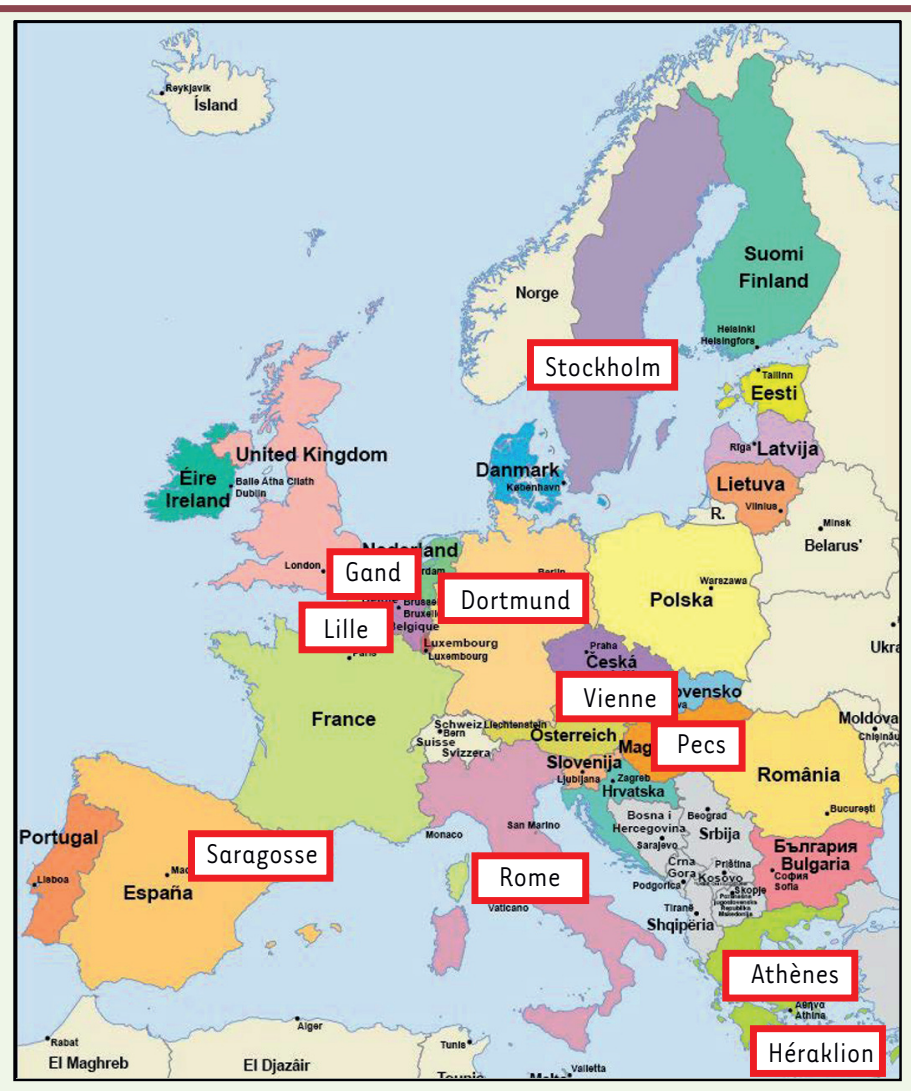

Figure 1. Villes participantes de l'étude HELENA entre 2006 et 2007.

conséquences possibles attribuables à leur conduite à l'âge adulte. Cette évaluation a pris en considération différentes composantes dont la mesure des apports alimentaires quotidiens, les connaissances et les habitudes nutritionnelles, les choix et les préférences alimentaires, la composition corporelle, l'évaluation de paramètres clinico-biologiques comme le statut vitaminique et immunitaire, l'activité et les performances physiques ainsi que des analyses génétiques centrées sur des gènes connus pour leur implication dans la corpulence et leurs interactions possibles avec la nutrition et l'environnement [4]. Toutes les techniques, tests et questionnaires qui ont été utilisés ont été validés. Un manuel rassemblant toutes les fiches d'instructions de chaque appareil utilisé, des tests et des questionnaires disponibles en plusieurs langues a été publié en 2013 [6]. Les adolescents qui ont été sélectionnés étaient âgés entre 12,5 et 17,5 ans. Ils étaient issus de 10 villes de plus de 100000 habitants réparties dans 9 pays d'Europe (Figure 1) [5]. Sur 5759 adolescents initialement contactés et informés, 3528 ont participé à l'étude et ont été retenus pour les analyses. Un tiers d'entre eux ont bénéficié de prises de sang pour des analyses de polymorphisme génétique, de dosages métaboliques, de vitamines et de micronutriments, et de marqueurs de l'inflammation.

Au-delà de la photographie complète de la population étudiée (une information qui permet de rendre compte des habitudes de vie à un temps t), l'objectif de ce projet était donc d'identifier des facteurs qui puissent être associés à l'état nutritionnel des sujets qu'ils soient individuels (polymorphismes génétiques, influence de l'entourage proche, etc.) ou, plus généralement, environnementaux comme l'aménagement du territoire urbain. Notre propos est de présenter les résultats qui ont été obtenus, du microscopique au macroscopique, c'est-à-dire de l'influence sur le statut de l'adolescent des gènes qu'il porte à l'influence de son environnement proche, et à plus grande échelle, à l'influence de son environnement urbain et de l'école (Figure 2).

\section{Facteurs individuels intrinsèques}

Les facteurs possiblement impliqués qui ont été étudiés en premier, sont les déterminants génétiques pouvant être associés à certaines pathologies. En effet, plusieurs polymorphismes génétiques (SNP : single nucleotide polymorphism) ont une influence néfaste sur le statut nutritionnel. Néanmoins, cette influence peut être «désactivée » par des facteurs extérieurs qui permettent de remédier aux aspects délétères dus à ces mutations. Ainsi, deux études ont concerné des gènes associés à l'IMC (index de masse corporelle) marqueur d'adiposité, une troisième s'est intéressée à un gène impliqué dans le métabolisme lipidique avec, comme marqueur, le taux de triglycérides sanguins, l'un des marqueurs du syndrome métabolique.

La première mutation intéresse le gène PPAR $Y 2$ (peroxysome proliferator activated receptor gamma 2). II s'agit de la substitution Prol2Ala (SNP rs1801282) qui est associée à une augmentation de la sensibilité à l'insuline avec les répercussions sur la corpulence qui en découle. Dans l'étude HELENA, $21 \%$ des adolescents étaient homozygotes pour cette mutation. Ils présentaient tous une tendance à avoir un IMC plus élevé que les adolescents sans mutation. Cependant, l'allaitement au cours de la petite enfance semble avoir un effet contrecarrant cette tendance. $\varepsilon n$ effet, les adolescents ayant la mutation et qui avaient été allaités avaient un IMC significativement plus faible que ceux ayant également la mutation mais qui n'avaient pas été allaités. En revanche, l'allaitement n'avait pas d'influence sur I'IMC des adolescents ne présentent pas la mutation. Cette observation confirme chez des adolescents présentant ce polymorphisme génétique, l'effet protecteur de l'allaitement maternel qui avait été observé antérieurement chez des nourrissons [7].

La seconde mutation qui a été étudiée porte sur le gène FTO (fat mass and obesity associated gene) 


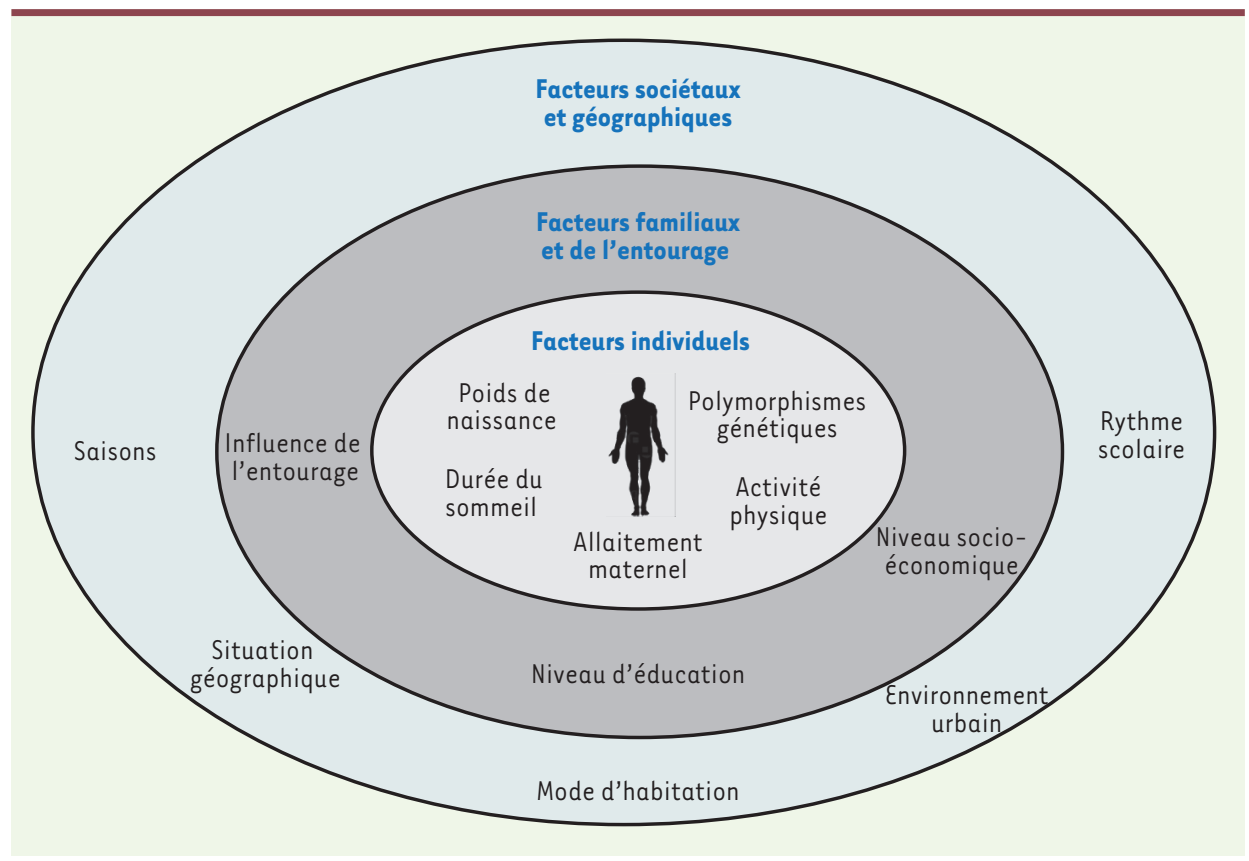

Figure 2. Les différents niveaux de facteurs influençant le statut nutritionnel des adolescents. poids de naissance et l'adiposité abdominale chez les adolescents ayant été allaités au moins 3 mois [11]. En revanche, aucune corrélation n'est observée chez les adolescents qui n'ont pas été allaités. Ces résultats montrent donc que les enfants nés avec un petit poids de naissance (< 1,5 kg) présentent plus de risque de développer une adiposité abdominale élevée à l'âge adulte et que l'effet du petit poids de naissance peut être atténué par l'allaitement maternel.

Enfin, la leptine, qui est une hormone impliquée dans la prise alimentaire, a été examinée [32] $(\rightarrow)$.

Elle est

(SNP rs9939609). Elle est connue pour sa répercussion néfaste sur I'IMC. Seize pour cent des adolescents de l'étude présentaient cette mutation. Sur la base de leur activité physique, mesurée par accélérométrie (Figure 3), les adolescents actifs (qui pratiquaient au moins $60 \mathrm{~min} /$ jour d'activité physique modérée à vigoureuse) et présentant la mutation avaient un IMC significativement plus faible que les adolescents présentant la mutation mais non actifs. Aucune différence d'IMC entre les actifs et les non actifs n'est observée chez les adolescents non mutés. Cette observation montre donc l'effet protecteur de la pratique d'activité physique particulièrement chez les adolescents présentant la mutation du gène FTO [8].

Enfin, la troisième mutation concerne le gène GCKR (glucokinase regulatory protein). La substitution Pro446Leu du gène (SNP rs1260326) est en effet associée à des conséquences néfastes sur le métabolisme lipidique. Trente et un pour cent des adolescents étudiés étaient homozygotes pour cette mutation. Considérant le taux de triglycérides mesuré chez ces adolescents, un effet protecteur de la consommation d'acides gras polyinsaturés oméga-3 a pu être mis en évidence alors qu'aucune différence n'est observée chez les adolescents ne présentant pas ce polymorphisme. Les acides gras polyinsaturés oméga-3 ont donc un effet protecteur chez les adolescents porteurs du polymorphisme du gène GCKR [9].

Dans une deuxième approche, l'étude a porté sur l'influence du poids à la naissance sur le développement de facteurs de risque à l'âge adulte. En effet, un petit poids de naissance est un risque accru à l'âge adulte de développer des désordres métaboliques comme la résistance à l'insuline et/ou une adiposité abdominale élevée [10]. Cet effet néfaste peut cependant être atténué, voire annulé, par l'allaitement ou la pratique d'une activité physique. L'analyse de l'adiposité abdominale des adolescents a révélé une corrélation inverse entre le en effet un marqueur de la résistance à l'insuline. Curieusement, chez les adolescents de l'étude,

$(\rightarrow)$ Voir la Nouvelle $m / s n^{\circ} 10$, octobre 2010, page 803 le taux de leptine est inversement corrélé au poids de naissance chez les filles, mais pas chez les garçons [12]. Cette corrélation négative entre leptine et poids de naissance chez les filles disparaît cependant chez les filles ayant une activité physique alors qu'elle persiste chez les filles inactives [13]. L'effet néfaste d'un petit poids de naissance sur certains paramètres métaboliques, en particulier le taux de leptine, peut néanmoins être atténué par une pratique d'activité physique.

\section{Influence des conditions de vie}

Une durée courte de sommeil augmente l'adiposité, le stress et altère les fonctions immunitaires [14]. Dans notre étude, l'influence de la durée du sommeil sur différents paramètres liés à l'adiposité, le stress ou le système immunitaire, a donc été examinée. Les adolescentes dormant moins de $8 \mathrm{~h}$ par jour ont présenté une masse grasse $7,8 \%$ plus importante que celles dormant plus de $8 \mathrm{~h}$. Une corrélation inverse entre durée du sommeil et taux sérique de cortisol, une hormone biomarqueur du stress, a également été observée aussi bien chez les garçons que chez les filles [15]. Finalement, l'influence néfaste d'une durée courte de sommeil sur le profil immunitaire, en particulier sur les taux de cytokines comme l'IL(interleukine)-5 (impliquée dans de C. Vatier et al., 


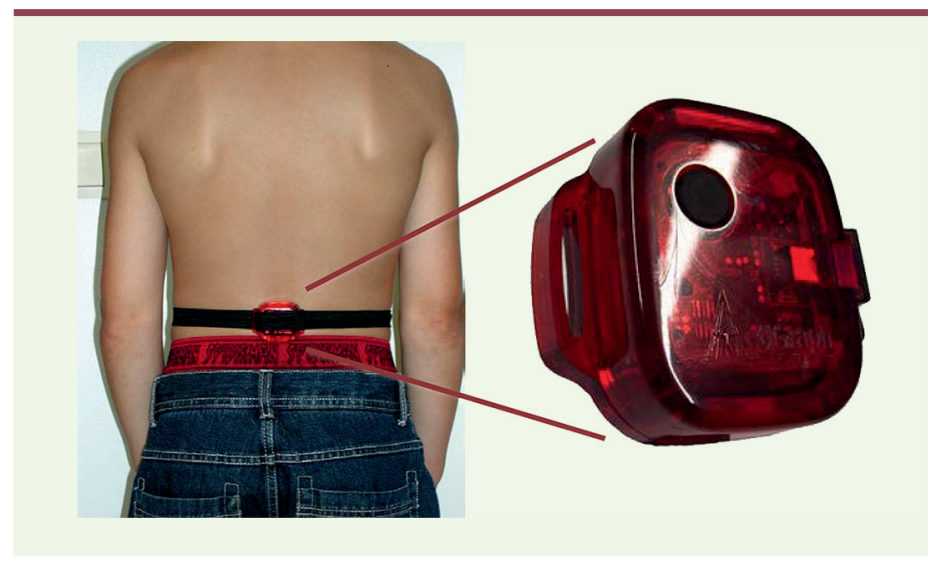

Figure 3. Évaluation de l'activité physique par accélérométrie pendant une semaine (Actigraph GTIX ${ }^{\circledR}$ ). Les données de l'accélomètre sont exprimées en nombre et intensité d'accélérations et décélérations par minutes (counts/ $\mathrm{min}$ ). Le sujet est considéré en activité modérée à vigoureuse lorsque les données sont au-dessus du seuil de 2000 counts/min (défini par des études précédentes réalisées en laboratoire).

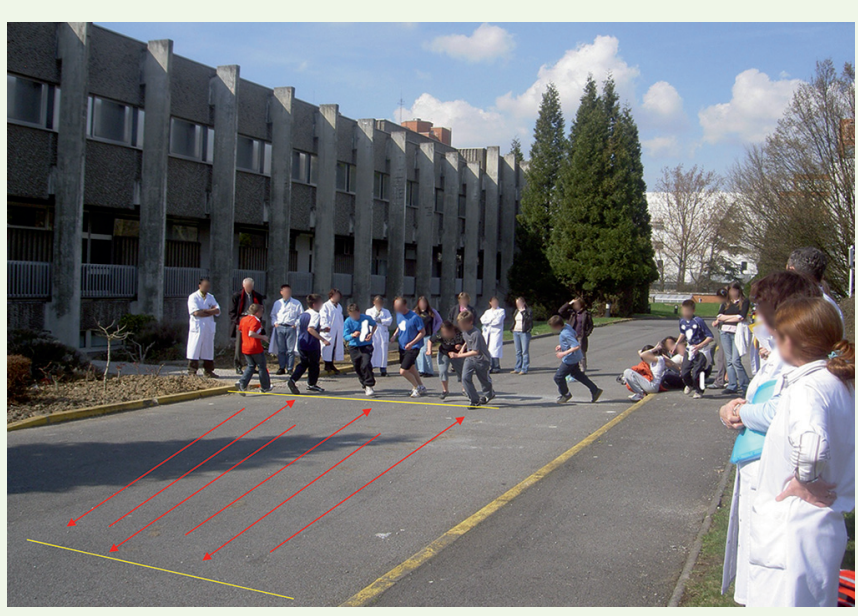

Figure 4. Mesure de l'endurance par évaluation indirecte de la $\mathrm{VO}_{2} \max :$ test $d u$ « shuttle run 》 du Docteur Léger. Les sujets courent de plus en plus vite (de $8 \mathrm{~km} / \mathrm{h}$ à leur vitesse maximale) selon une séquence imposé par un rythme sonore (à chaque «bip », le sujet doit se trouver sur la ligne jaune).

l'asthme et l'hyperéosinophilie) et l'IL-6 (pro-inflammatoire), a également été observé mais uniquement chez les filles.

Les effets bénéfiques de l'activité physique sont reconnus dans de nombreux domaines avec un seuil établi à $60 \mathrm{~min} /$ jour d'activité modérée à vigoureuse. Dans notre étude, les adolescentes actives présentent une densité minérale osseuse légèrement supérieure $(+4,4 \%)$ aux non-actives [16]. De façon intéressante, l'amélioration de la densité minérale osseuse sous l'action de la vitamine $D$ n'a été retrouvée que chez les adolescentes actives [17]. Chez ces adolescentes, une influence du niveau d'activité physique a également été observée sur le taux de leptine et d'insuline indiquant un effet favorable de l'activité physique sur le statut hormonal de l'adolescente [18]. Les adolescents actifs ont également une meilleure endurance cardiorespiratoire (de $+11 \%$ mesurée par la $\left.\mathrm{VO}_{2} \max \right)$ [19]. Ces adolescents actifs qui ont une bonne endurance cardiorespiratoire (Figure 4), ont également les scores d'inflammation les plus faibles [20].

Ainsi, les adolescents actifs ont une meilleure condition physique (Figures 4 et 5 : endurance cardiorespiratoire et force des membres supérieurs) qui est corrélée à des facteurs favorables de bonne santé comme la diminution de l'inflammation [7, 21-23], de l'insulinorésistance [18] et de la graisse abdominale [24].

\section{Influence de l'entourage}

Le niveau socio-économique des parents peut influencer le statut des adolescents. En effet, nous avons observé dans notre groupe d'adolescents une corrélation positive entre le niveau socio-économique des parents et la pratique d'activité physique des enfants [25]. Néanmoins, il est à noter que, quel que soit leur statut socioéconomique, les parents qui encouragent leurs enfants à pratiquer une activité physique constatent généralement une augmentation de leur niveau de performance. Une augmentation de 10,4\% du niveau de l'activité physique des enfants est ainsi observée lorsque les encouragements proviennent du père [26]. Mais lorsque les encouragements sont prodigués par la mère, le niveau d'activité physique n'augmente pas. L'influence du père semble donc plus importante.

Concernant la qualité de l'alimentation il semble que l'importance soit donnée à la mère. La qualité de l'alimentation évaluée par le DQI (diet quality index), un indice prenant en compte la diversité de l'alimentation, son équilibre, sa richesse en fruits et légumes [27], montre en effet que c'est la mère qui a le plus d'influence. Les adolescents qui reçoivent de forts encouragements de leur mère à s'alimenter sainement, ont un DQI supérieur de 5,1\% par rapport à celui des adolescents qui n'ont aucun encouragement. À l'inverse, les encouragements à manger sainement qui proviennent du père, des frères et sœurs ou d'amis ne modifient pas le DQI (données personnelles de Vanhelst et al., 2016).

\section{Facteurs sociétaux et géographiques}

Les rythmes scolaires sont différents à travers l'Europe. Ils peuvent être distingués en cycle court (avec une fin des cours dans la journée avant $15 \mathrm{~h}$ ) et en cycle long (avec une fin des cours après $15 \mathrm{~h}$ ). Les adolescents ayant un rythme scolaire court ont un 

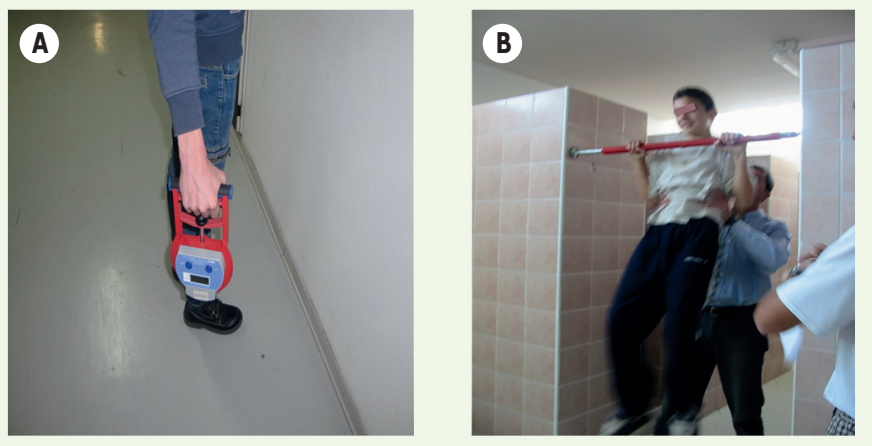

Figure 5. Évaluation de la force des membres supérieurs. A. Le sujet serre la poignée du dynamomètre le plus possible. $\boldsymbol{B}$. Le sujet reste en suspension menton au-dessus de la barre le plus longtemps possible.

niveau d'activité physique hebdomadaire diminué de 7,8\% par rapport aux adolescents du cycle long (données personnelles de Vanhelst et al, 2016). Ceci peut s'expliquer en partie par le fait que dans le cycle long, il est proposé plus de temps de récréation ( $55 \%$ de durée en plus) pendant le temps scolaire, avec un niveau d'activité physique à ce moment augmenté de $43 \%$. Les adolescents ayant un rythme de cycle court passent, en dehors des périodes de cours, $106 \mathrm{~min} / \mathrm{j}$ en moyenne de plus en sédentarité que les adolescents du cycle long. II apparaît donc que les adolescents sont physiquement actifs lors des récréations dans le cadre scolaire mais qu'ils restent sédentaires en dehors.

L'influence des saisons sur l'activité physique est minime. En effet, il n'existe pas de différence globale de niveau d'activité physique suivant les saisons. La seule différence est observée chez les filles qui sont plus sédentaires en hiver (10 min par jour en plus) [28]. En revanche, il existe une différence de niveau d'activité physique entre les adolescents du nord de l'Europe (Allemagne, Autriche, Belgique, France, Hongrie et Suède) et ceux du sud (Espagne, Grèce, Italie). Les garçons du nord font en effet $7,3 \%$ d'activité physique de plus par rapport à ceux du sud. Ce taux atteint $16,2 \%$ de plus pour les filles [29]. Une différence entre nord et sud est également retrouvée en ce qui concerne la qualité de l'alimentation évaluée par le DQI [27] puisque les adolescents du sud ont un DQI $13 \%$ supérieur à celui des adolescents du nord. De façon intéressante, le DQI des adolescents du nord augmente de $16 \%$ si le niveau d'éducation de la mère est haut, ce qui n'est pas le cas chez les adolescents du sud, pour lesquels le DQI n'est pas influencé par la mère. Ainsi, les mères de l'Europe du nord qui ont un niveau d'éducation élevé proposent une alimentation de meilleure qualité à leurs adolescents.

L'environnement urbain influence également l'activité physique des adolescents : ainsi ceux vivant dans un environnement avec beaucoup de trafic routier passent moins de temps dans les activités physiques par rapport à ceux qui vivent dans un environnement moins urbain (3\% en moins). L'environnement urbain a également une influence sur la condition physique (Figures 4 et 5). L'endurance cardiorespiratoire est en effet diminuée chez les adolescents vivant dans un environnement avec beaucoup de trafic. À l'inverse, la présence de pistes cyclables, de trottoirs, parcs et jardins, et de salles de sports proches du domicile améliore tous les paramètres de la condition physique [30].

\section{Conclusion}

Les résultats de cette large étude révèlent l'influence de nombreux paramètres individuels, collectifs et d'environnement sur le statut nutritionnel, le niveau de l'activité et la condition physique des adolescents (Figure 2). L'étude HELENA trouve sa puissance dans le nombre élevé de sujets qui ont été évalués, I'utilisation de techniques de référence validées, et dans une évaluation très large du statut nutritionnel intégrant des facteurs cliniques, biologiques, comportementaux et d'environnement. Cependant, sa principale limite est liée à son caractère observationnel, méthodologie qui ne permet que de montrer l'existence de possibles associations mais pas de liens de cause à effet. L'une des principales perspectives de ce travail est la mise en place du suivi longitudinal des adolescents étudiés afin de connaître leur devenir. Ceci sera réalisé dans le cadre d'une future étude, BELINDA (better life by nutrition during aging), représentant ainsi une formidable opportunité d'identifier des facteurs influençant, à l'occasion du passage de l'adolescence à l'âge adulte, le risque cardiovasculaire en particulier. $\diamond$

\section{SUMMARY}

From the influence of genes to the influence of family and urban environment on the nutritional status, activity, and physical condition of european urban adolescents

HELENA was a cross-sectional study carried out from 2006 to 2007 in more than 3500 adolescents aged from 12.5 to 17.5 years old through 10 Europeans towns from 9 countries. Its objective was to assess adolescent nutritional status including: body composition, biological markers, physical activity and fitness. This study shown the high impact of socio-economic condition, life style and personal and collective environment, dietary pattern (including breastfeeding), some genetic mutations involved in adiposity and metabolism, physical activity level and fitness on adolescent nutritional status. $\diamond$

\section{REMERCIEMENTS}

Les auteurs remercient Jacques Isaert, dessinateur du CHRU de Lille pour les illustrations.

L'étude HELENA a été financée par la Communauté Européenne (Sixth RTD Framework Programme : Contract FOODCT-2005-007034) et pilotée à partir de 2006 par un consortium européen rassemblant 18 universités ou instituts de recherche et 8 industriels de l'agroalimentaire. 


\section{LIENS D'INTÉRÊT}

Les auteurs déclarent n'avoir aucun lien d'intérêt concernant les données publiées dans cet article.

\section{RÉFÉRENCES}

1. Lissau I, Overpeck MD, Ruan WJ, et al. Body mass index and overweight in adolescents in 13 European countries, Israel, and the United States. Arch Pediatr Adolesc Med 2004 ; 158 : 27-33.

2. Bass $S$, Delmas PD, Pearce $G$, et al. The differing tempo of growth in bone size, mass, and density in girls is region-specific. J Clin Invest $1999 ; 104: 795-804$.

3. Lobstein T, Frelut ML. Prevalence of overweight among children in Europe. Obes Rev $2003 ; 4$ : 195 200

4. Moreno LA, Gonzalez-Gross M, Kersting M, et al. Assessing, understanding and modifying nutritional status, eating habits and physical activity in European adolescents: the HELENA (healthy lifestyle in Europe by nutrition in adolescence) Study. Public Health Nutr $2008 ; 11: 288-99$.

5. Beghin L, Castera M, Manios Y, et al. Quality assurance of ethical issues and regulatory aspects relating to good clinical practices in the HELENA cross-sectional study. Int J Obes (Lond) 2008 ; 32 Suppl $5:$ S12-8

6. Gonzalez-Gross M, De Henauw S, et al. Manual of operation the HELENA study. Saragosse (Espagne) : Presses Universitaires de Saragosse $2013: 289$ p.

7. Verier $C$, Meirhaeghe A, Bokor S, et al. Breast-feeding modulates the influence of the peroxisome proliferator-activated receptor-gamma (PPARG2) Prol2Ala polymorphism on adiposity in adolescents The healthy lifestyle in Europe by nutrition in adolescence (HELENA) cross-sectional study. Diabetes Care 2010 ; 33 : 190-6.

8. Ruiz JR, Labayen I, Ortega FB, et al. Attenuation of the effect of the FTO rs 9939609 polymorphism on total and central body fat by physical activity in adolescents The HELENA study. Arch Pediatr Adolesc Med $2010 ; 164: 328-33$.

9. Rousseaux J, Duhamel A, Dumont J, et al. The $n-3$ long-chain PUFAs modulate the impact of the GCKR Pro446Leu polymorphism on triglycerides in adolescents. J Lipid Res $2015 ; 56: 1774-80$.

10. Barker DJ. The developmental origins of adult disease. J Am Coll Nutr $2004 ; 23$ : 588S-595S.

11. Labayen I, Ortega FB, Ruiz JR, et al. Breastfeeding attenuates the effect of low birthweight on abdominal adiposity in adolescents: the HELENA study. Matern Child Nutr 2015 ; 11 : 1036-40.

12. Labayen I, Ruiz JR, Huybrechts I, et al. Sexual dimorphism in the early life programming of serum leptin levels in European adolescents: The HELENA study. J Clin Endocrinol Metab 2011 ; 96 : ع1330-६4.

13. Labayen I, Ortega FB, Moreno LA, et al. Physical activity attenuates the negative effect of low birth weight on leptin levels in European adolescents; The HELENA study. Nutr Metab Cardiovasc Dis $2013 ; 23: 344-9$.

14. Faraut B, Boudjeltia KZ, Vanhamma L, Kerkhofs M. Immune, inflammatory and cardiovascular consequences of sleep restriction and recovery. Sleep Med Rev $2012 ; 16$ : 137-49.

15. Perez de Heredia F, Garaulet M, Gomez-Martnez S, et al. Self-reported sleep duration, white blood cell counts and cytokine profiles in European adolescents: the HELENA study. Sleep Med 2014 ; 15 : 1251-8.

16. Gracia-Marco L, Moreno LA, Ortega FB, et al. Levels of physical activity that predict optimal bone mass in adolescents The HELENA study. Am J Prev Med 2011 ; 40 : 599-607.

17. Valtuena J, Gracia-Marco L, Vicente-Rodriguez G, et al. Vitamin D status and physical activity interact to improve bone mass in adolescents. The HELENA study. Osteoporos Int $2012 ; 23: 2227-37$.

18. Jimenez-Pavon D, Castillo MJ, Moreno LA, et al. Fitness and fatness are independently associated with markers of insulin resistance in European adolescents; the HELENA study. Int J Pediatr Obes $2011 ; 6: 253-60$.
19. Martinez-Gomez D, Ruiz JR, Ortega FB, et al. Recommended levels and intensities of physical activity to avoid low-cardiorespiratory fitness in European adolescents: the HELENA study. Am J Hum Biol 2010 ; 22 : $750-6$.

20. Artero EG, Espana-Romero V, Jimenez-Pavon D, et al. Muscular fitness, fatness and inflammatory biomarkers in adolescents. Pediatr Obes $2014 ; 9$ : $391-400$.

21. Martinez-Gomez D, Gomez-Martinez S, Ruiz JR, et al. Objectively-measured and self-reported physical activity and fitness in relation to inflammatory markers in European adolescents: the HELENA Study. Atherosclerosis 2012; $221: 260-7$.

22. Ferrari $M$, Cuenca-Garcia $M$, Valtuena J, et al. Inflammation profile in overweight/obese adolescents in Europe: an analysis in relation to iron status. Eur J Clin Nutr $2015 ; 69: 247-55$.

23. Vyncke K, Cruz FE, Fajo-Pascual M, et al. Validation of the diet quality Index for adolescents by comparison with biomarkers, nutrient and food intakes: the HELENA study. Br J Nutr $2013 ; 109: 2067-78$

24. Moliner-Urdiales D, Ruiz JR, Ortega FB, et al. Association of objectively assessed physical activity with total and central body fat in Spanish adolescents: the HELENA study. Int J Obes (Lond) 2009 ; 33 : 1126-35.

25. De Cocker K, Artero EG, De Henauw S, et al. Can differences in physical activity by socio-economic status in European adolescents be explained by differences in psychosocial correlates? A mediation analysis within the HELENA (healthy lifestyle in Europe by nutrition in adolescence) study. Public Health Nutr $2012 ; 15: 2100-9$.

26. Martin-Matillas M, Ortega FB, Ruiz JR, et al. Adolescent's physical activity levels and relatives physical activity engagement and encouragement: the HELENA study. Eur J Public Health $2011 ; 21$ : 705-12.

27. Beghin L, Dauchet L, De Vriendt T, et al. Influence of parental socioeconomic status on diet quality of European adolescents: results from the HELENA study. Br J Nutr 2014 ; 111 : 1303-12.

28. Gracia-Marco L, Ortega FB, Ruiz JR, et al. Seasonal variation in physical activity and sedentary time in different European regions. The HELENA study. J Sports Sci 2013 ; 31 : 1831-40.

29. Ruiz JR, Ortega FB, Martinez-Gomez D, et al. Objectively measured physical activity and sedentary time in European adolescents: the HELENA study. Am J Epidemiol $2011 ; 174: 173-84$.

30. Vanhelst J, Beghin L, Salleron J, et al. A favorable built environment is associated with better physical fitness in European adolescents. Prev Med $2013 ; 57: 844-9$.

31. Vanasse A, Demers M, Hemiari A, Courteau J. Obésité, malbouffe et sédentarité : des inséparables? Med Sci (Paris) 2007 ; $23: 5-6$.

32. Vatier C, Capeau J, Vigouroux C. La leptine, nouvelle perspective thérapeutique dans le traitement du diabète? Med Sci (Paris) $2010 ; 26$ : 803-7.

\section{TIRÉS À PART}

L. Beghin

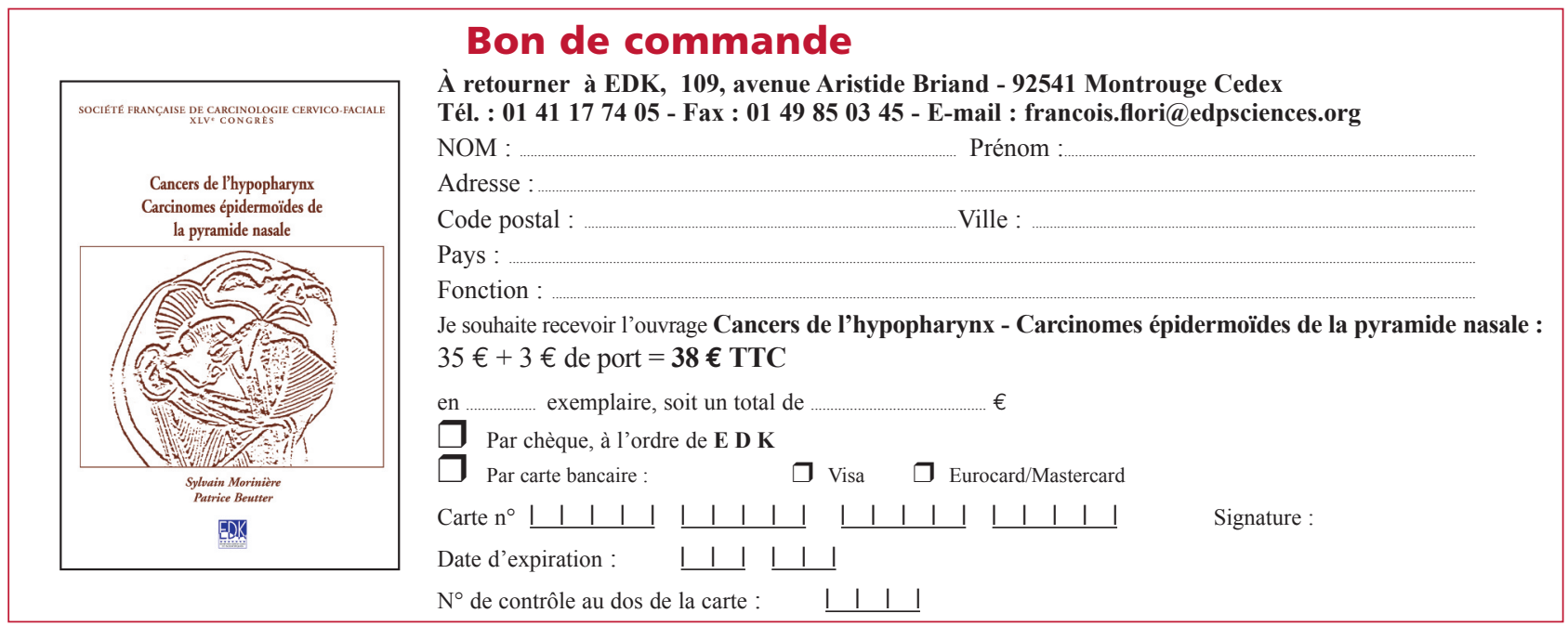

